\title{
Benefits of a High Speed Low Point Spread Detector For Monochromated Electron Energy-Loss Spectroscopy
}

\author{
Liam Spillane $^{1 *}$, Andrew Yankovich ${ }^{2}$, Paolo Longo ${ }^{1}$, Ray D. Twesten ${ }^{1}$, Eva Olsson ${ }^{2}$ \\ 1. Gatan Inc., Pleasanton, CA 94588, USA \\ 2. Chalmers University of Technology, Department of Physics, Gothenburg, Sweden \\ * Corresponding author: 1spillane@gatan.com
}

Indirect electron detectors suffer from inherent point spread - information from a single electron strike is spread over multiple detector channels which has the effect of limiting the achievable energy resolution for electron energy-loss spectroscopy (EELS). The effect of point spread is stronger at high microscope accelerating voltage and low spectrometer energy dispersion. Spectral features at sub $1 \mathrm{eV}$ energy loss in the electron energy loss spectrum typically demonstrate weak scattering intensity and are in close proximity to the zero loss peak. While high dispersion low $\mathrm{kV}$ conditions give optimal detector conditions for high energy resolution data acquisition, a non-monochromated zero loss peak is typically spread over a sufficient number of detector channels to make analysis in the sub $1 \mathrm{eV}$ energy loss region impossible.

Due to desire to perform anaylsis in this energy range, considerable interest and effort has been directed toward advancing column technology to drastically reduce the energy spread in the scanning transmission electron microscope. Recent improvements in electron column and monochromator design have enabled the achievement of energy resolutions of better than $10 \mathrm{meV}$ so that analysis at ultra low energy loss and in particular, vibrational EELS, is now a reality [1,2]. Previous studies on boron nitride have shown that the main phonon peak is resolvable at $15-28 \mathrm{meV}$ energy resolution at $60 \mathrm{kV}[1,3]$. While much effort has been invested in improving energy resolution by reducing energy spread of the electron beam, less attention has been give to detector optimisation. Sub $1 \mathrm{eV}$ analysis is typically performed at low accelerating voltage and high dispersion to minimise the effect of point spread. Perfoming analysis at low $\mathrm{kV}$ and high dispersion presents a number of challenges. The energy range that may be studied is small, limiting field of view and complicating processing steps desirable for low loss such as log deconvolution and Kramers-Kronig analysis [4]. Spectral intensity is also weaker than at low dispersion, making peak detection more challenging and necessitating longer exposure times, increasing specimen dose. The use of a low beam energies also places higher constraints on specimen thickness and while the knock on damage threshold is reduced compared to high $\mathrm{kV}$, radiolytic damage increases.

Low loss electron energy-loss spectra have been acquired from a broad selection of materials using a new high speed indirect electron detector and next generation energy filter. All data was acquired using a monochromated electron beam with energy $40-200 \mathrm{kV}$. Preliminary results for boron nitride are shown in figure 1. The increasing effect of point spread with increased acceleration voltage is clear. The zero loss and phonon peaks in figure 1(a) are less narrow than the equivalent peaks in figure 1(b) and figure 1(c). While broadening was observed with increasing beam energy, it is also clear that the boron nitride main phonon peak was resolvable at all conditions due to the low point spread of the detector used. In this presentation the advantages of using a low point spread detector for high energy resolution low loss analysis including: vibrational, band gap and plasmon peak characterisation will be demonstrated. 
[1] O. L. Krivanek, et al., Nature (London) 514, 209 (2014)

[2] T. Miyata, et al., Mizoguchi, Microscopy 63, 377 (2014)

[3] R. Nicholls, et al., Microsc. Microanal. 21 (suppl 3), (2015)

[4] J. A. Alexander, et al. Ultramicroscopy 180 (2017)
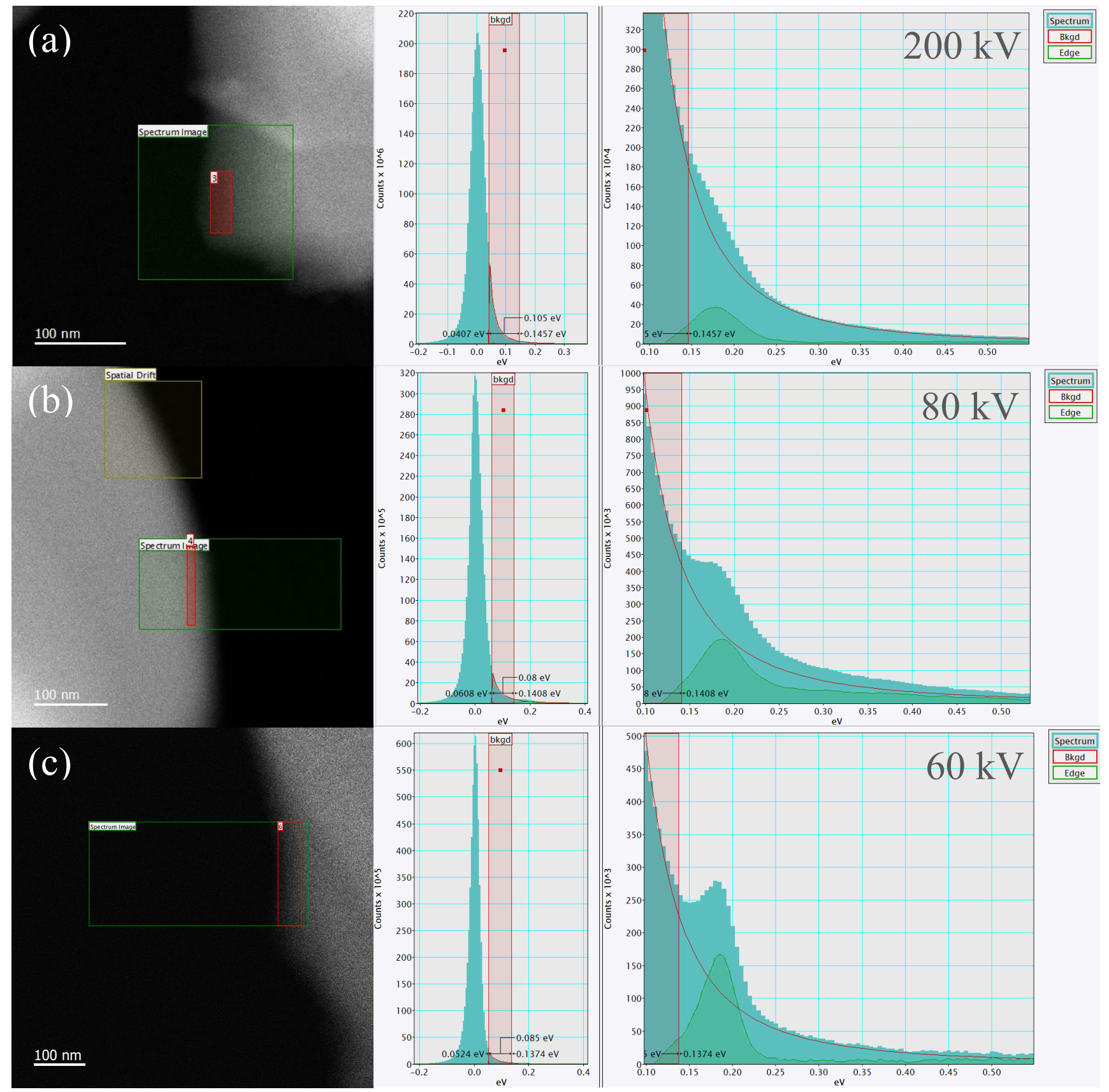

Figure 1. EELS spectrum images acquired from bulk boron nitride. Spectra shown to the right hand side are extracted and summed over the red regions highlighted on the ADF survey images to the left. The data shown was acquired at: (a) $200 \mathrm{kV}$, (b) $80 \mathrm{kV}$ and (c) $60 \mathrm{kV}$. The main boron nitride phonon peak is shown in all cases. 